"This is the peer reviewed version of the following article: Hill, G. and Kemp, S. M. (2016), Uh-Oh! What Have We Missed? A Qualitative Investigation into Everyday Insight Experience. The Journal of Creative Behavior. doi: 10.1002/jocb.142, which has been published in final form at DOI: 10.1002/jocb.142. This article may be used for noncommercial purposes in accordance with Wiley Terms and Conditions for Self Archiving. "

\title{
Uh-oh! What Have We Missed? A Qualitative Investigation into Everyday Insight Experience
}

\section{Gillian Hill and Dr. Shelly M. Kemp}

University of Buckingham

Running Head: Everyday experience of insight

Contact: Gillian Hill. Department of Psychology. University of Buckingham. Hunter Street.

|Buckingham. MK18 1EG. United Kingdom

E-mail: gillian.hill@buckingham.ac.uk

Submission Date: December $3^{\text {rd }} 2014$

Revised Submission Date: May $18^{\text {th }} 2015$

Second Revision Submitted: October 21st 2015

Accepted: December 9th 2015 


\begin{abstract}
This study takes a novel qualitative approach to the investigation of everyday insight experience. It offers ecological validation to findings principally rendered, prior to this research, from a quantitative, cognitive standpoint. In addition, it considers emotional as well as cognitive components of insight. Participants were given different (or no) definitions of insight to ensure experiences collected did not simply mirror the examples of insight provided. This avoided the circularity problem of previous insight research. With the use of an open-ended questionnaire (online or hardcopy), first-hand textual accounts of insight instances were recorded. Data collected from 76 participants was analysed using an adapted qualitative methodology, Integrative Thematic Analysis. This enabled the researchers to identify themes from the data, building a new typology of insight: Content (Personal, Intellectual, Practical), Process (Social Facilitation, Time Away, Active Search) and Feelings (Positive Feelings, Negative Feelings) aspects of insight. The findings suggest everyday experience of insight reaches beyond cognitive problem solving to include elements related to applied psychology, namely Personal (counselling psychology) and Social Facilitation (occupational psychology). Notably, this study offers examples of negative insight, Uh-oh moments, for the first time. Future research should focus on the interaction of cognitive and affective components in insight moments.
\end{abstract}

Keywords: insight, everyday experience, Aha moment, Uh-oh moment, integrative thematic analysis 


\section{Uh-oh! What Have We Missed? A Qualitative Investigation into Everyday Insight Experience}

Insight has been defined quite specifically in relation to problem solving events as a sudden solution moment usually described as being accompanied by an emotional feeling of Aha (see review by Weisberg, 2014). Others provide more general descriptions of insight such as the experience of a new thought or understanding, without constraining it to instances of problem solving (e.g., Klein \& Jarosz, 2011). While affective aspects are often alluded to in reference to Aha or Eureka moments, the focus of insight research has neglected to investigate this emotional component and in the main has concentrated on cognitive processes. Perhaps due to the unpredictable nature of insight the prevailing approach to its study has been to artificially elicit insight moments under controlled conditions.

Consequently, there is little research into naturalistic insight experiences (Chu \& MacGregor, 2011; Jarman, 2014). This study aims to address these issues and investigate evidence for the existing assumptions about insight using qualitative research methods.

\section{Circularity Problem in Insight Research}

Gestalt psychology introduced the experimental notion of insight describing it as sudden cognitive restructuring. Defining it as such constrained its subsequent investigation to being an exclusively cognitive phenomenon and has inherently influenced the types of tasks used to elicit insight. This has led to a circularity problem of insight research, where narrow definitions are seen to lead to narrow research results (Dominowski and Dallob, 1995; Ollinger and Knoblich, 2009).

Weisberg (2014) suggests that insight be considered beyond a problem solving paradigm. One potential alternative case of insight, 'discovered insight problem solving', was identified by Csikszentmihalyi and Sawyer (1995) through analysis of interview transcripts with prominent creative individuals. They concluded that insight was not just pinpointed to 
the moment of solving a clearly defined problem, but that it may also serve as the initiation to problem solving. Casting insight as a problem finding process in addition to problem solving can be seen to contradict most notions of insight and highlights one aspect that might have been overlooked in respect of insight. This study therefore aimed to explore if insight would be described in relation to other parts of the problem solving process in addition to the solution moment. Giving different definitions to different participants (some receiving a description making explicit cognitive reference to the problem solution moment, whilst others did not receive a problem solving prompt) provided the opportunity to demonstrate that participant descriptions were a true reflection of people's insight experiences rather than a simple primed response to the definition given.

A further omission from current conceptions is negative insight. Gick and Lockhart (1995) discuss a possible example of this in chagrin, a feeling of annoyance (negative emotion) at having been tricked or at missing something obvious. This is demonstrated in popular culture by the cartoon character Homer Simpson's 'D'oh' that accompanies his negative insight moment on suddenly understanding something patently obvious to others. Gick and Lockhart (1995) discuss a further cartoon example in the context of humour and insight: Although they do not explicitly identify it as such, Gary Larsson's comic cartoon is an example of a different kind of negative insight. It pictures an aeroplane cockpit with two pilots who are questioning the presence of a goat in the clouds in front of them; the humour is found in the pilots' lack of insight into the impending collision with the unseen mountain (under the goat!). Such insight from the pilots' perspective would not be seen with a positive emotion such as humour or a negative 'D'oh' moment (a positive reaction to having solved the goat riddle, followed by annoyance at it having been obvious). It would more likely be a purely negative response; a sudden realisation accompanied by an Uh-oh moment, which 
could be considered as an antonym to the Aha moment more often discussed in terms of insight.

\section{Everyday Experience of Insight}

Klein and Jarosz (2011) described their study as giving naturalistic evidence of insight by analysing real-life incidents from historical or anecdotal reports. They included cases where a person made a "radical shift" (Klein \& Jarosz, 2011, p. 338) in his or her mental model that was towards an improved or more useful representation (adaptive function of insight). However, the reliance on historical events is problematic due to subjectivity of interpreting second hand reports. Additionally, retrospective accounts may be exposed to many renditions in which memory refinements are made (Gruber, 1995). These issues cast doubt on how representative their findings really are of real life insight experience.

The study of insight in ecologically valid contexts is an emerging field of research (Jarman, 2014). One aspect relating to this is the prevalence of insight in everyday life, which Ohlsson (2011) highlights is unknown, but likely rare. Further to this, attempts that have been made to investigate naturalistic experience of insight use selective samples. For example, Csikszentmihalyi and Sawyer (1995) and Klein and Jarosz (2011) take a "Great Minds Approach" (Sternberg \& Davidson, 1995, p. 327). This method focuses on processes that occur in highly creative individuals, with the assumption that key characteristics of their insight could be generalised to the population.

The "Great Minds" described in insight research might be seen as akin to Big-C creativity which focuses on celebrated examples of creativity. Big-C is distinguished from little-c every day creativity (seen in the lay person). A further useful distinction is that of Kaufman and Beghetto (2009) who extend the Big-C/ little-c model. Firstly they differentiate between eminent Big-C creativity not seen as an everyday life occurrence (for instance, Albert Einstein) and the daily Pro-c of creative, yet non-eminent professionals (e.g., fashion 
designers and professional chefs). Hargadon and Bechky (2006) and Dunbar (1995) investigated insight in such Pro-c samples, respectively focusing on teams of creative consultants (e.g. in product design) and creative scientific discovery. Kaufman and Beghetto (2009) also identify mini c, an additional form of every day personal creativity, where individuals' "intrapersonal insights" (p. 4) represent moments that are creative purely from the individual's own perspective. Fundamentally, an approach selecting participants for their creative attributes (Big-C and Pro-c) neglects the role of insight across these other aspects of everyday life (little-c, and mini-c).

Consequently, this study aimed to capture first hand, self-reported textual accounts of insight experiences through an open-ended questionnaire, minimising memory bias by asking for recent experiences of insight (within the last 24 hours). The use of open-ended questionnaires are beneficial because of the larger number of participants they enable relative to other qualitative methods such as face to face, semi-structured interviews. Also, it allowed a novel approach for qualitative research, by which participants were grouped and offered different working definitions of insight between questionnaires to compare responses given and account for the possibility of primed responses. The qualitative analysis was undertaken from a realist perspective, which could be argued to be more superficial than phenomenological approaches. However, it was felt to be appropriate as it is unlikely that a lay perspective of insight involves the deep philosophical and personal deliberation that might be seen for example when considering a person's conception of their own illness. As this study was exploratory in nature, it aimed to draw conclusions on how wider aspects of insight such as affective components might be experienced in everyday life and described by lay participants not selected for any creative characteristics.

\section{Method}

\section{Participants}


Participants were a snowball sample recruited through promotion (via email to colleagues and on the researchers' social media pages) of links to the online questionnaire. A hard copy version of the questionnaire was also distributed to associates of the researchers both within the university and local community. Seventy-six participants (21 male and 55 female) completed the questionnaire (an additional 77 individuals started the online questionnaire but withdrew before completion). Participants were both students (abbreviated to $S$ in results, 27 women, 10 men, $M_{\text {age }}=27.3$ years, $S_{\text {age }}=8.95$, age range: $18-53$ years) and non-students (abbreviated to NS in results, 28 women, 11 men, $\mathrm{M}_{\mathrm{age}}=42.9, \mathrm{SD}_{\mathrm{age}}=$ 13.69, age range: $21-74$ years). All participants gave informed consent and approval for the study was obtained from the relevant University ethics committee.

\section{Materials and Procedure}

A questionnaire was designed for use in Survey Monkey or in hard copy. On giving informed consent and providing demographic information (age, country of main residence/birth and occupation), first time participants were randomly allocated to one of three groups. Each group received a different definition page (either on screen or in the paper version), all containing an image of a matchstick figure with a light bulb above its head. One had no accompanying definition (No Definition, abbreviated as ND in results). A second contained a cognitive, problem solving focused definition: "insight is a sudden solution to a long vexing problem, a sudden recognition of a new idea, or a sudden understanding of a complicated situation accompanied by an Aha moment" (Jung-Beeman, Collier \& Kounios, 2008, p.20) (abbreviated as C in results). The third group were given a less prescriptive cognitive definition without explicit reference to problem solving: "Insight is a change in mindset or shift in thinking" (Klein and Jarosz, 2011, p. 338) with the additional instruction that, "it is accompanied by an Aha moment for a positive realisation or an Uh-oh moment when it is negative" (Emotional definition, abbreviated as E in results). 
All participants were initially asked if they had experienced insight in the last twentyfour hours. The majority of participants indicated that they had and completed an Insight Log page to record their experience. Each insight was captured in open text responses to the following questions: Provide a brief description of your insight experience? What were you doing when you experienced this insight? What were your thoughts leading to the insight? Additionally, participants were asked to indicate: Approximately how long have you been trying to solve this problem? They responded selecting from options; minutes, hours, days, weeks, months, years or my insight wasn't a solution to a problem. Finally, participants were debriefed and invited to return in the future to share any further experiences of insight. Return participants $(n=2)$ were not grouped or offered insight definitions, but directed straight to an Insight Log page on giving their consent and demographic information (participants were advised that this information would be used to map return responses to original ones).

Participants responses were generally quite brief, ranging from seven words, for example describing their insight as being "what to do for a party [whilst] meditating" to 150 words (total words across their responses to all the questions described above). A high number of potential participants $(n=77)$ did not complete the online questionnaire. Many withdrew at the point when asked to give qualitative responses sharing their experiences of insight. Initially participants were unable to complete the questionnaire without giving experience details. This was amended midway through data collection by adding the following response options: I have never had an insight; I have had insights but cannot remember them; I do not want to share my insight.

\section{Analysis of data}

Braun and Clarke (2006) describe the stages of thematic analysis as: i) familiarisation of data; ii) tentatively labelling and grouping the excerpts; iii) searching for themes among the groups identified; iv) reviewing the themes; v) defining and naming the themes. 
Typically, one researcher conducts all these stages. This may sometimes be presented to a second researcher for verification. In light of circularity issues, bias following this methodology can be introduced by a first researcher's previous knowledge of the literature. This may be further confounded by confirmation bias of the second researcher's verification.

To reduce bias, in this study two independent researchers both separately conducted the first three stages and identified candidate themes/sub-themes. Then they shared their themes and definitions with one another and identified concurrent themes which were labelled and the definitions refined to provide a final typology. This Integrative Thematic Analysis (ITA) is a novel adaptation of methods described by Braun and Clarke (2006), aiming to provide greater objectivity among the final themes identified. Table 1 demonstrates the application of ITA by the two researchers (R1 and R2) in this study and their identified themes.

\section{Results}

The final integrated themes identified by using ITA, are proposed by the researchers to represent different aspects of insight, specifically the Content, Process and Feelings (Figure 1). For example, a participant may report an insight that is of Intellectual Content solved during a Process of Active Search and is accompanied by Positive Feelings. The themes and their groupings into superordinate aspects seen in the typological model in Figure 1 and described below, result from the later stages of ITA and the process in which the two researchers integrated their themes. Below are the definitions and descriptions of the themes the researchers identified with supporting excerpts. These were selected after avoiding those that could have been in direct response to the definition given. Excerpts are labelled with abbreviations, defined in the methods, describing participants' sex, age, student/non-student status and definition group (No Definition, Cognitive or Emotional).

\section{Content [Intellectual/Practical/Personal]}


The Content aspect of insight relates to the context of the described experience in three distinct problem solving domains: Intellectual, Practical or Personal problems.

Intellectual. Participants' described solving theoretical problems mainly of a scientific or conceptual basis. For example, "I was reading an article at the time, and a particular phrase struck me. I suddenly realised that there was a connection between the subject I was reading up about, and my more usual area of study" (F, 53, NS, E). The wide support for the Intellectual theme could be argued to originate in the student participants, however as demonstrated by the previous excerpt non-students also provided support for this.

Practical. Several insight logs described problems requiring physical manipulation on objects. For instance, a problem such as "how to use tyre air pressure pump at garage for the first time, could not work out how to attach it to tyre valve [and] suddenly realised how to attach it after examining the device" (F, 41, S, E).

Personal. Many participants' described insight experiences relating to reflection on their personal lives or relating to their mind set. These Personal insight experiences were, in quite general terms, often relating to how they planned to change. For instance, one participant identified the wide-ranging insight of the "power of focusing on one thing in life, and all else will fall into place automatically" (M, 24, S, E). Some however, reflected a specific event or aspect of the participant's life; as illustrated by the insight that "I can't make my wedding perfect. Sometimes I need to save my energy and accept a compromise solution earlier" (F, $35, \mathrm{NS}, \mathrm{ND})$.

\section{Process [Active Search/Time Away/Social Facilitation]}

The Process aspect represents a description of how participants' arrived at their insight. Three different processes to solve problems were identified: Active search, Time Away and Social Facilitation. Participants' volunteered process accounts within their insight 
descriptions, not only when asked what they were doing at the time. As such they can be considered valid themes.

Active Search. One process for problem solving that was most often described under Intellectual Content problems was Active Search. Participants' describe the nature of effort in their attempt to solve a problem, often by reading or thinking specifically about the problem until reaching a solution. One participant describes his effort to perfect a magic trick.

Sometimes the method behind a trick requires an awkward body position that, if not justified within the context of the performance, makes the method obvious to the audience. In this situation I need to keep my arm bent in a certain way. I realized that my arm looks totally natural in that position if I am holding something up to my face, as if trying on a pair of glasses. It just suddenly made sense. (M, 28, S, C)

Time Away. Several participants' described being engaged in activities unrelated to the insight they had, often occurring whilst lying in bed, in a relaxed state or driving. One participant described, "I had been thinking hard about what I had to do, but then my mind wandered. During the wandering, I realised the solution to my problem" (F, 20, S, E). In contrast to the many longer Time Away examples offered, here the Time Away can be seen to be a very short mental break from effortful problem solving.

An additional strand of reports demonstrate insights resulting in an improved solution to a problem already solved, therefore they represent Time Away solutions in the sense that the participant was not actively searching as they already had a solution, but happened (through insight) upon a better one. For instance one participant reports that they "realised that there was a better way of manipulating a psychological construct. Happily I was in the process of designing a study so hadn't already started collecting data" (F, 27, NS, E). 
Social Facilitation. Insights were described by many participants as occurring as a result of a social interaction either in a teaching context or through discussion. Implicit in the identification of this theme is the contrasting circumstance where there is no social facilitation, seeing insight within the individual, rather than in a social framework. In a teaching context, one participant explains, "someone was explaining how something worked, and I didn't understand, until it suddenly clicked and it made perfect sense" (F, 23, NS, C).

\section{Feelings [Positive Feelings/Negative Feelings]}

The Feelings aspect represents themes describing the specific emotional context of the insight descriptions and accordingly incorporates the themes of Positive Feelings and Negative Feelings. This is the aspect where there is least agreement between the researchers' initial interpretations of the data. While both identified positive and negative feelings in relation to insight, the focus of these was different. However, while the researchers independently focused on different aspects of feelings, the later stages of the ITA process found consensus on the overarching themes of Positive Feelings and Negative Feelings as best representing the data.

Positive Feelings. The first researcher focused on more general affect expressed towards the insight. Their candidate theme, Certainty, describes positive affective responses to insight experiences. Some participants' described having a sense of intuition, being sure they were onto the right answer before having an insight. Others expressed their assurance that the answer was correct in terms of positive emotion post insight. Both intuition and certainty are reflected in the following insight description.

It was a crystallisation of a research problem I'd been thinking about [ ] before I had traced the whole path, I suddenly knew where it was going, and really had this feeling of crystallisation, of a definite form emerging. Though initially 
indistinct, I knew it was what I needed for the research problem to move on a step. $(\mathrm{F}, 35, \mathrm{~S}, \mathrm{C})$

The second researcher did not so much pick up on the positive affect surrounding certainty of an insight but identified participants' more general positive or optimistic outlook on life expressed in their insight descriptions. The above quote illustrates that these two conceptions are not mutually exclusive, as the participant's expression can be seen as generally upbeat as well as positive in terms of certainty

Negative Feelings. Both researchers' identified instances where a participant's realization was negative. Whilst none explicitly referred to an Uh-oh feeling, they can be interpreted as likely to have had such a response due to the undesirable nature of the insight. For instance describing, "driving home when I suddenly realised that I hadn't saved the essay draft I'd worked on all day, as I had downloaded it from my [online cloud file] and worked on it in uni [versity]. So I realised I had lost a whole days work" (M, 21, S, C). It is apparent that this participant's sudden realisation was a negative experience likely accompanied by some utterance, either out loud or in the mind along the line of oh-no!

The second researcher additionally identified stress as being implicitly implied in some insight reports and this was thought to have had negative connotations to the experience leading up to the insight moment. For instance one participant describes their insight occurring whilst, "crying while trying to sleep" (F, 18, S, C).

\section{Discussion}

This study provides, for the first time, examples of insight experienced in the everyday life of a sample not selected for their creative characteristics. It also demonstrates original adaptions to typical qualitative methods, firstly in grouping participants to account for potential priming effects of definitions given. Secondly, the use of ITA enabled the 
researchers to identify a typological model of insight (Figure 1) from the data with interrelated themes: Feelings (Positive Feelings and Negative Feelings), Content (Personal, Practical and Intellectual) and Process (Social Facilitation, Time Away and Active Search). The themes Practical, Intellectual, Active Search and Time Away compliment findings from the predominant quantitative approach to the study of insight, so adding an ecologically valid foundation for this body of research. Findings also expand the construct of insight to incorporate applied aspects from outside of the creativity domain. The Personal theme is related to counselling psychology with possible links to the "intrapersonal insight" (Kaufman \& Beghetto, 2009, p. 4) seen in mini-c creativity. Social Facilitation may relate to occupational psychology and the study of creativity as a group process (Csikszentmihalyi \& Sawyer, 1995). Notably, the theme of Negative Feelings encompasses a novel aspect of insight with the first recording of Uh-oh moments. Moreover, negative insight may occur at a different point and serve a different function in the creative problem solving process than positive insight. As such our study provides a number of interesting potential research avenues that will likely broaden our conception of insight and creative problem solving.

\section{Feelings}

Negative Feelings. Negative insight has not been considered before within insight research other than in cursory theoretical discussion (Gick and Lockhart, 1995). This study suggests that insight be seen as a sudden and adaptive cognitive change accompanied by an affective component that can be negative (an Uh-oh moment) as well as positive. A new understanding about a situation might be the sudden realisation that things are worse than originally thought. This can be distinguished from Chagrin (Gick and Lockhart, 1995) that describes negative emotions of regret or annoyance at having missed an obvious insightful solution, rather than a negative response to the adverse content of the insight. 
The negative insight serves to initiate the problem solving process rather than conclude it, providing motivation to solve the problem (Runco, 1994). This is of course adaptive, as the first step to dealing with a problem is recognising that it exists. Such a perspective echoes Csikszentmihalyi and Sawyer's (1995) "discovered insight problem solving" (p. 337) and likewise is consistent with the notion of problem finding posited by Runco and Chand (1995) as a primary skill in creative thinking. This highlights that insight might occur earlier in the process than proposed by traditional problem solving models (such as Wallas, 1926).

Positive Feelings. Positive aspects of insight as identified in this study are often discussed in relation to Aha moments within insight literature, but swiftly overlooked in terms of experimental exploration. As such, this study contributes real-life examples to the emerging body of research, which aims to capture phenomenological descriptions of Aha moments (Danek et al., 2014; Jarman, 2014).

One stream of experimental research which does focus on feelings in relation to insight captures participants' 'feelings of warmth' (how close they felt they were to a solution) whilst problem solving (Metcalfe \& Weibe, 1987). While this might be attributed to feelings it could also be related to metacognition: The problem solver's thoughts about their proximity to the solution rather than their emotional responses to insight (Weisberg, 1992). This aligns with the positive feelings of certainty identified by the first researcher.

\section{Content}

Personal. The Personal theme sits distinctly outside the traditional cognitive approach to the study of insight, mapping more closely to counselling psychology literature. Within this domain, in both the psychoanalytic and humanistic traditions, self-insight is positioned as a contributing element enabling an individual to recognise the root of their difficulties and effect therapeutic change (Connolly et al., 1999; McLeod, 2013; Williams \& Lynn, 2010). Many of the Personal insights shared in this study can be seen as equivalent to Hillis et al.'s 
(2015) 'Stimulating Mastery'; using self-understanding (or understanding about others in relation to one's self) to respond to problems in everyday life.

Furthermore, Moro, Avidibegovic and Moro (2012) highlight that insight in psychotherapy is a process that enables change. In this sense, Personal insight falls at the beginning of the problem solving process. Timulak and McElvaney (2013) likewise found that therapy clients described how their insight gave clarity to their problems rather than representing an absolute solution. This is consistent with the examples of Uh-oh suggesting that real life occurrences of insight are not constrained to solution moments.

Personal insight identified here can additionally be seen to correspond to the "intrapersonal insights" (Kaufman \& Beghetto, 2009, p. 4) that exemplify mini-c creativity. Kaufman \& Beghetto (2009) describe mini-c in terms of learning in children and adults (relating to the Practical/Intellectual themes discussed below). This theme highlights a potential extension to their conception incorporating insight experiences related to counselling psychology. The personal realisations reported in this study meet the widely applied criteria for creativity of being novel and useful when considered from the perspective of the individual experiencing them (Plucker, Beghetto \& Dow, 2004).

Practical/ Intellectual. The Practical and Intellectual themes relate much more closely to experimental cognitive insight research. The two themes echo distinctions sometimes made by researchers when categorising classic insight problems: for instance, conceptual, verbal and mathematical problems (emerged here as Intellectual) are typically differentiated from spatial problems. The latter is identified as Practical in this study because participants' described manipulating objects in the real world (e.g. Dow and Mayer, 2004; Gilhooly \& Murphy, 2005).

\section{Process}


Time Away / Active Search. The Process themes of Active Search and Time Away are also seen to closely align with cognitive psychology and are particularly pertinent to discussions regarding the necessity of impasse in insight. Examples of the Time Away theme can be seen to corroborate the possible role of impasse and incubation in everyday experience of insight (Ohlsson, 2011, 1992; Sio \& Omerod, 2009; Baird et al., 2012). Recent studies suggest the biggest positive impact in problem solving success occurs with incubation tasks of low cognitive load (Baird et al., 2012; Sio and Omerod, 2009). Many of the examples offered in this study fit such a description with insights occurring whilst walking, running or driving. Some might argue that the identified theme Time Away is equivalent to incubation, but this research aims to distinguish between the two. Incubation, as recognised by previous research implies substantial time away from a problem (Sio and Ormerod, 2009), whilst the newly identified theme of Time Away is broader and incorporates shorter time periods away. This corroborates suggestions that such brief mind wandering, often colloquially termed daydreaming, might be related to creative problem solving (Schooler et al., 2011).

The second Process theme, Active Search, however suggests insight also occurs whilst participants are actively searching for a solution: So without the necessity of impasse posited by Ohlsson (1992) and in line with findings from verbal protocol analysis of insightful problem solving (Fleck \& Weisberg, 2004, 2013).

Social Aspects. Social aspects of insight have previously been highlighted, often in workplace studies (Csikszentmihalyi \& Sawyer, 1995; Dunbar, 1995; Hargadon \& Bechky, 2006). This study identifies a similar aspect in every day experience of insight and again confounds the notion that insight be narrowly conceived in terms of the individual. Future studies should consider moving away from the paradigm of individual participants' completing laboratory-based problem solving tasks.

Many of the socially induced insights described a teaching context. As highlighted 
above, Kaufman \& Beghetto (2009) identify insights occurring during learning as examples of mini-c creativity. The teaching examples shared in this study support their distinction of mini-c from little-c. The insights described would clearly not be new or creative when considered from the perspective of the teacher, so not really fulfilling the requirement of little-c as being an everyday creative experience. However, from the perspective of the individual having the insight, it would be novel and useful, an intrapersonal experience of creativity. Likewise, insights where there is no social facilitation but where participants describe them as being the result of individual study or practice of a skill, for example perfecting a magic trick, would also qualify as mini-c.

\section{Limitations and Future Directions}

Many of the insight examples collected in this study are open to subjective interpretation as the researchers were unable to probe responses given due to the online questionnaire design. While participants were encouraged to provide as full an explanation of their insight experience as possible, in reality descriptions were often short. The opportunity to probe participants regarding their insight logs would enable much finer grained analysis of their experience, reducing subjectivity and subsequently enabling more confident comparison to other research. Such limitations could be addressed using follow-up semi structured interviews. However, this would in turn have limitations in terms of the number of participants it would be practicable to include and the reduction in anonymity that an online questionnaire affords.

Furthermore, as an exploratory qualitative study, an element of interpretation is an integral part of the process. The development of ITA aimed to increase the objectivity of the analysis through verification between two researchers' identifying concurrent themes. One of the researchers being naïve to the prior body of insight research additionally protected against the circularity problem of insight research. The introduction of participant grouping, likewise 
reduced circularity effects. Many excerpts from the no definition group reassuringly supported all themes identified.

A further limitation to note may relate to the high dropout rate of participants observed at the point where they were asked to describe their insight. While it may be due to genuine withdrawal from the study, it is also conceivable that participants were unable to recall an insight experience, despite the majority of these cases indicating initially that they had experienced recent insight. It would therefore be desirable to follow participants more closely, asking them to capture their insight experiences in real time to eliminate any memory effects. This might be achieved in a diary study using ecological momentary assessment (Stone and Shiffman, 1994). Furthermore, such methodology would enable subsequent probing of their responses to elicit rich detailed accounts of every day experience of insight. Additionally, following participants in real-time may give some indication of the prevalence of insight. This is identified by Ohlsson (2011) as a yet unknown, but desirable detail that is likely to contribute to our future conceptions of insight.

Whilst this study highlights an emotional aspect of insight, no conclusions can be drawn as to how and when the 'thinking' and 'feeling' components interact. Future research would likely explore this by applying current theories that integrate emotion and cognition to insight. This would provide questions to experimentally test and work towards a conceptual model of insight incorporating both affective and cognitive perspectives. For example, exploring insight from a basic emotions approach (Ekman \& Friesen, 1971; Ekman, Sorenson and Friesen, 1969). The basic emotions of happiness and surprise have been discussed with respect of insight and Aha moments. It is less clear, however what discrete negative emotion might be seen in an Uh-oh moment. Future work could look to clarify the roles of negative emotions such sadness, fear, disgust and anger in insight. 
An alternative could be to take a dimensional approach to emotion in insight, rather than look to classify associated discrete emotions which some argue constrains research (Ellsworth, 2014). From this perspective, models that incorporate both cognitive and affective components may be useful. Gross (2015) for instance, has introduced valuations of valance in his recently proposed Extended Process Model of Emotion Regulation. He suggests that on perception of an internal world event, a "good for me/ bad for me" (p. 10) evaluation is made. As insight is an internal world event, Gross' (2015) theory could provide a process explanation as to how an insight is seen as an Aha (good for me) or an Uh-oh (bad for me) moment.

Whichever theoretical approach to is taken to emotion, experimental tasks will need to be developed that elicit the full repertoire of insight experiences in order to test derived hypotheses. Current methods only generate problem solving, Aha moments (see recent reviews for summaries of insight tasks, including Batchelder and Alexander, 2012; Chu and MacGregor, 2011; Dietrich \& Kanso, 2010). Therefore, future work will need to incorporate negative insight.

\section{Conclusion}

This study provides a first step to the naturalistic exploration of insight in the everyday lives of ordinary people. The use of qualitative methodology, in this case ITA, can be seen as complimentary to experimental work in the study of insight. This highlights the value of adopting a mixed methods approach in future research. The current study's resultant themes offer tentative support for the validation of experimental research and confound potential criticisms relating to the circularity problem. Additionally, this study demonstrates the potential to strengthen links between insight research founded in theoretical and applied fields. Finally, the findings have highlighted the need for more exploration of affective 
aspects of insight, in particular the consideration of negative insight in the form of Uh-oh moments. 


\section{References}

Baird, B., Smallwood, J., Mrazek, M. D., Kam, J. W., Franklin, M. S. \& Schooler, J. W. (2012). Inspired by distraction mind wandering facilitates creative incubation. Psychological Science, doi: 10.1177/0956797612446024

Batchelder, W. H., \& Alexander, G. E. (2012). Insight problem solving: A critical examination of the possibility of formal theory. The Journal of Problem Solving, 5(1), 6. doi: $10.7771 / 1932-6246.1143$

Braun, V. \& Clarke, V. (2006). Using thematic analysis in psychology. Qualitative research in psychology, 3(2), 77-101. doi: 10.1191/1478088706qp063oa

Chu, Y. \& MacGregor, J. N. (2011). Human performance on insight problem solving: A review. The Journal of Problem Solving, 3(2), 6. Retrieved from Ebsco Discovery

Connolly, M. B., Crits-Christoph, P., Shelton, R. C., Hollon, S., Kurtz, J., Barber, J. P., ... \& Thase, M. E. (1999). The reliability and validity of a measure of self-understanding of interpersonal patterns. Journal of Counseling Psychology, 46(4), 472. doi: 10.1037/0022-0167.46.4.472

Csikszentmihalyi, M. \& Sawyer, K. (1995). Creative insight: The social dimension of a solitary moment. In R. J. Sternberg \& J. E. Davidson (Eds.), The nature of insight (pp.329-363). Cambridge, MA: MIT Press

Danek, A. H., Fraps, T., Von_müller, A., Grothe, B. \& Öllinger, M. (2014). It's a kind of magic-what self-reports can reveal about the phenomenology of insight problem solving. Theoretical and Philosophical Psychology, 5, 1408. doi: 10.3389/fpsyg.2014.01408

Dietrich, A., \& Kanso, R. (2010). A review of EEG, ERP, and neuroimaging studies of creativity and insight. Psychological bulletin, 136(5), 822. doi: 10.1037/a0019749 
Dominowski, R.L. \& Dallob, P. (1995) Insight and problem solving. In R. J. Sternberg, J. E. Davidson (Eds.), The nature of insight (pp. 33-62). Cambridge, MA US: The MIT Press

Dow, G. T. \& Mayer, R. E. (2004). Teaching students to solve insight problems: Evidence for domain specificity in creativity training. Creativity Research Journal, 16, 389-402

Dunbar, K.1995. How scientists really reason: Scientific reasoning in real-world laboratories, Sternberg RJ, Davidson JE (Eds.), The nature of insight (pp 365-395). Cambridge, MA US: The MIT Press.

Ekman, P., \& Friesen, W. V. (1986). A new pan-cultural facial expression of emotion. Motivation and emotion, 10(2), 159-168. doi: 10.1007/BF00992253

Ekman, P., Sorenson, E. R., \& Friesen, W. V. (1969). Pan-cultural elements in facial displays of emotion. Science, 164(3875), 86-88. doi: 10.1126/science.164.3875.86

Ellsworth, P. C. (2014). Basic emotions and the rocks of New Hampshire. Emotion Review, 6(1), 21-26.doi: 10.1177/1754073913494897

Fleck, J. I. \& Weisberg, R. W. (2004). The use of verbal protocols as data: An analysis of insight in the candle problem. Memory \& Cognition, 32(6). doi: 990-1006 10.3758/BF03196876

Fleck, J. I. \& Weisberg, R. W. (2013). Insight versus analysis: Evidence for diverse methods in problem solving. Journal of Cognitive Psychology, 25(4), 436-463. doi: $10.1080 / 20445911.2013 .779248$

Gick, M. L. \& Lockhart, R. S. (1995). Cognitive and affective components of insight. In R. J. Sternberg, J. E. Davidson (Eds.), The nature of insight (pp. 197-228). Cambridge, MA US: The MIT Press.

Gilhooly, K. J. \& Murphy, P. (2005). Differentiating insight from non-insight problems. Thinking \& Reasoning, 11(3), 279-302. doi: 10.1080/13546780442000187 
Gross, J. J. (2015). Emotion regulation: Current status and future prospects. Psychological Inquiry, 26(1), 1-26. doi: 10.1080/1047840X.2014.940781

Gruber, H. E. (1995). Insight and affect in the history of science. In R. J. Sternberg, J. E. Davidson (Eds.), The nature of insight (pp. 397-431). Cambridge, MA US: The MIT Press.

Hargadon, A. B. \& Bechky, B. A. (2006). When collections of creatives become creative collectives: A field study of problem solving at work. Organization Science, 17(4), 484-500. doi: 10.1287/orsc. 1060.0200

Hillis, J. D., Leonhardt, B. L., Vohs, J. L., Buck, K. D., Salvatore, G., Popolo, R., \& ... Lysaker, P. H. (2015). Metacognitive reflective and insight therapy for people in early phase of a schizophrenia spectrum disorder. Journal of Clinical Psychology, 71(2), 125-135. doi: 10.1002/jclp.22148

Jarman, M. S. (2014). Quantifying the qualitative: measuring the insight experience. Creativity Research Journal, 26(3), 276-288. doi: 10.1080/10400419.2014.929405

Jung-Beeman, M., Collier, A. \& Kounios, J. (2008). How insight happens: learning from the brain. NeuroLeadership Journal, 1(1), 20-5. Retrieved from http://www.neuroleadership.com/research/journal

Kaufman, J. C., \& Beghetto, R. A. (2009). Beyond big and little: The four c model of creativity. Review of general psychology, 13(1), 1-12 doi: 10.1037/a0013688

Klein, G. \& Jarosz, A. (2011). A naturalistic study of insight. Journal of Cognitive Engineering and Decision Making, 5(4), 335-351. doi: 10.1177/1555343411427013

McLeod, J. (2013). An introduction to counselling. Maidenhead: McGraw-Hill.

Metcalfe, J. \& Wiebe, D. (1987). Intuition in insight and noninsight problem solving. Memory \& Cognition, 15, 238-246. doi: 10.3758/BF03197722 
Moro, L., Avdibegović, E. \& Moro, I. N. (2012). Insight in psychotherapy. Psychiatria Danubina, 24(3), 46-47.

Ohlsson, S. (1992). Information-processing explanations of insight and related phenomena. In: M. Keane and K. Gilhooly (eds.), Advances in the Psychology of Thinking. (pp. 144). London: Harvester-Wheatsheaf,

Ohlsson, S. (2011). Deep learning: How the mind overrides experience. Cambridge University Press.

Ollinger, M. \& Knoblich, G. (2009). Psychological research on insight problem solving. In: H. Atmanspacher \& H. Primas (ed.) Recasting Reality (pp. 275-300). Berlin. Springer

Plucker, J. A., Beghetto, R. A., \& Dow, G. T. (2004). Why isn't creativity more important to educational psychologists? Potentials, pitfalls, and future directions in creativity research. Educational Psychologist, 39(2), 83-96. doi: 10.1207/s15326985ep3902_1

Runco, M. A. \& Chand, I. (1995). Cognition and creativity. Educational Psychology Review, 7(3), 243-267. doi: 10.1007/BF02213373

Runco, M. A. (1994). Problem finding, problem solving, and creativity. Norwood, NJ: Ablex.

Schooler, J. W., Smallwood, J., Christoff, K., Handy, T. C., Reichle, E. D. \& Sayette, M. A. (2011). Meta-awareness, perceptual decoupling and the wandering mind. Trends in cognitive sciences, 15(7), 319-326. doi: 10.1016/j.tics.2011.05.006

Sio, U. N. \& Ormerod, T. C. (2009). Does incubation enhance problem solving? A metaanalytic review. Psychological bulletin, 135(1). doi: 94.10.1037/a0014212

Sternberg, R. J. \& Davidson, J. E. (Eds.), The nature of insight. Cambridge, MA US: The MIT Press.

Stone, A. \& Shiffman, S. (1994) Ecological momentary assessment (EMA) in behaviorial medicine. Annals of Behavioral Medicine, 16:199-202. 
Timulak, L. \& McElvaney, R. (2013). Qualitative meta-analysis of insight events in psychotherapy. Counselling Psychology Quarterly, 26(2), 131-150. doi: $10.1080 / 09515070.2013 .792997$

Wallas, G. (1926). The art of thought. NY, USA: Harcourt Brace.

Weisberg, R. W. (1992). Metacognition and insight during problem solving: Comment on Metcalfe. Journal of Experimental Psychology: Learning, Memory, and Cognition, 18(2), 426-431. doi: 10.1037/0278-7393.18.2.426

Weisberg, R. W. (2014). Toward an integrated theory of insight in problem solving. Thinking \& Reasoning, (ahead-of-print), 1-35. doi: 10.1080/13546783.2014.886625

Williams, J. C. \& Lynn, S. J. (2010). Acceptance: An historical and conceptual review. Imagination. Cognition and Personality, 30(1), 5-56. doi: 10.2190/IC.30.1.c 
Table 1. ITA process demonstrating independently identified themes / sub-themes that lead to final integrated themes.

\begin{tabular}{|c|c|c|c|c|c|}
\hline & \multirow[t]{2}{*}{ Theme* } & \multirow[t]{2}{*}{ Sub-Theme* } & \multicolumn{3}{|c|}{ Final Theme ${ }^{* *}$} \\
\hline & & & Content & Process & Feelings \\
\hline \multirow[t]{5}{*}{$\mathrm{R} 1$} & $\begin{array}{l}\text { Self } \\
\text { Realisation }\end{array}$ & & Personal & & \\
\hline & Social Aspects & & & $\begin{array}{l}\text { Social } \\
\text { Facilitation }\end{array}$ & \\
\hline & $\begin{array}{l}\text { Problem } \\
\text { Solving }\end{array}$ & & $\begin{array}{l}\text { Intellectual } \\
\text { Practical }\end{array}$ & & \\
\hline & & $\begin{array}{l}\text { Active Search } \\
\text { Time Away }\end{array}$ & & $\begin{array}{l}\text { Active Search } \\
\text { Time Away }\end{array}$ & \\
\hline & Feelings & $\begin{array}{l}\text { Certainty } \\
\text { Uh-oh }\end{array}$ & & & $\begin{array}{l}\text { Positive } \\
\text { Negative }\end{array}$ \\
\hline \multirow[t]{5}{*}{$\overline{\mathrm{R} 2}$} & $\begin{array}{l}\text { Life } \\
\text { Reflections }\end{array}$ & $\begin{array}{l}\text { Positive } \\
\text { Affirmations } \\
\text { Change/Self } \\
\text { Improvement }\end{array}$ & $\begin{array}{l}\text { Personal } \\
\text { Personal }\end{array}$ & & $\begin{array}{l}\text { Positive } \\
\text { Negative }\end{array}$ \\
\hline & $\begin{array}{l}\text { Theoretical/ } \\
\text { Conceptual }\end{array}$ & $\begin{array}{l}\text { Trying Hard/ } \\
\text { Stress } \\
\text { Relaxed/Quiet } \\
\text { Time }\end{array}$ & $\begin{array}{l}\text { Intellectual } \\
\text { Intellectual }\end{array}$ & $\begin{array}{l}\text { Active Search } \\
\text { Time Away }\end{array}$ & $\begin{array}{l}\text { Negative } \\
\text { Positive }\end{array}$ \\
\hline & Visual-Spatial & & Practical & & \\
\hline & $\begin{array}{l}\text { One-Solution } \\
\text { Problems }\end{array}$ & & $\begin{array}{l}\text { Intellectual } \\
\text { Practical }\end{array}$ & & \\
\hline & $\begin{array}{l}\text { Learning/ } \\
\text { Being Taught }\end{array}$ & & & $\begin{array}{l}\text { Social } \\
\text { Facilitation }\end{array}$ & \\
\hline
\end{tabular}

* ITA stage iii - Candidate Themes / Sub-Themes ** ITA stage iv \& v 
Figure 1. Final model of typology of insight identified using Integrated Thematic Analysis. Themes interact in three aspects: Content, Process and Feelings. Both dark grey and light grey cubes demonstrate themes with distinct supporting excerpts in this study. Light grey cubes highlight novel aspects identified by this research. Dark grey cubes demonstrate the predominant focus of previous experimental approaches. White cubes did not have strong support from the excerpts in this study but provide conceptually possible categories for future research to investigate.

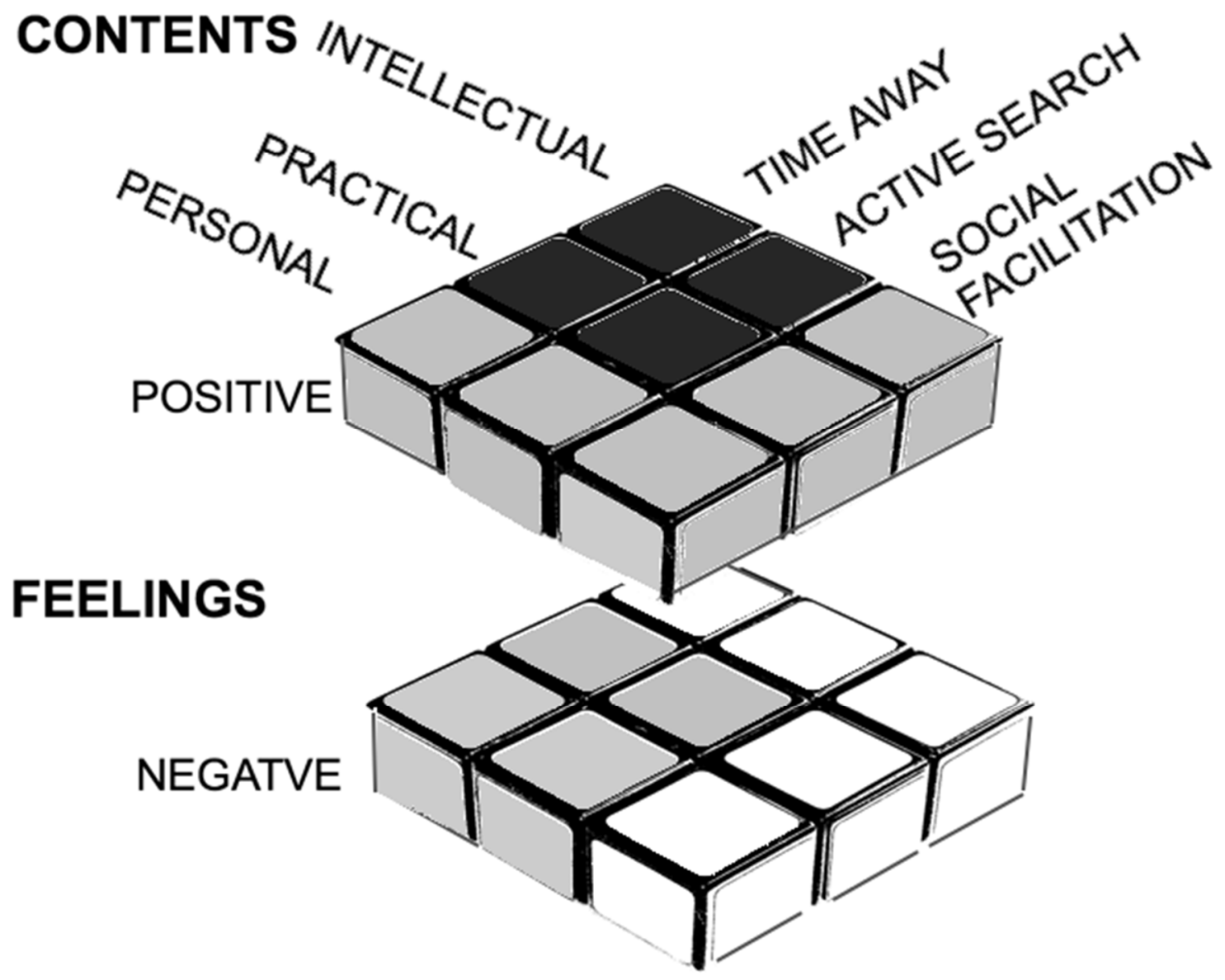

